\title{
Automated HF Modelling of Induction Machines Considering the Effects of Aging
}

\author{
Riccardo Leuzzi \\ Department of Electrical and \\ Information Engineering \\ Politecnico di Bari \\ Bari, Italy \\ riccardo.leuzzi@poliba.it \\ Pericle Zanchetta \\ Department of Electrical and \\ Electronic Engineering \\ University of Nottingham \\ Nottingham, UK \\ pericle.zanchetta@nottingham.ac.uk
}

\author{
Vito Giuseppe Monopoli \\ Department of Electrical and \\ Information Engineering \\ Politecnico di Bari \\ Bari, Italy \\ vitogiuseppe.monopoli@poliba.it
}

\author{
Francesco Cupertino \\ Department of Electrical and \\ Information Engineering \\ Politecnico di Bari \\ Bari, Italy \\ francesco.cupertino@poliba.it
}

\begin{abstract}
The use of wide bandgap semiconductor devices, such as $\mathrm{SiC}$ and GaN MOSFETs, in high-frequency converters introduces new challenges for the design of electric drives. The very fast switching transient of which these devices are capable, in fact, can become a serious threat for the reliability of the entire system. Electromagnetic interferences due to the high $\mathrm{dv} / \mathrm{dt}$ and $\mathrm{di} / \mathrm{dt}$, voltage reflections along the cable that cause overvoltage and ringing at the motor terminals, and large common-mode voltages that produce current circulation in the motor bearings are recognized as the major phenomena leading to premature failure of the drive. It is therefore important for designers to approach the problem from a system point of view, having the possibility to accurately model the system in the highfrequency domain to take appropriate measures to increase reliability. In this paper, an automated fitting procedure is proposed to identify the high-frequency model of an induction machine, which is based on using a genetic optimization algorithm to find the best rational approximation for the motor characteristics. A series of accelerated electrical aging tests have also been performed on the motors. The results are used iteratively in the proposed fitting procedure to obtain a timevarying model taking into account the aging progression.
\end{abstract}

Keywords-AC machines, automatic modelling, electrical aging, genetic algorithm, high-frequency behavior.

\section{INTRODUCTION}

The use of fast wide bandgap semiconductor devices, such as $\mathrm{SiC}$ MOSFETs, in high-frequency (HF) power electronic systems appears to be a consolidated solution to drive highspeed electrical machines, which require elevated control and switching frequencies to be stably operated [1], [2]. This trend is also driven by the reduced switching losses that such devices exhibit with respect to the previous silicon technology, since higher conversion efficiency is obtainable and smaller heat dissipation systems are required, fully exploiting the benefits of high speed in terms of weight, volume and cost reduction [3]. However, both the fast voltage slew rate $(\mathrm{dv} / \mathrm{dt})$ produced by modern MOSFETs and the elevated switching frequency they operate are among the major causes of reliability reduction in electrical drives. Three are the main phenomena affecting the reliability of the drive that are enhanced by the fast switching of modern devices. On the one hand, the large $\mathrm{dv} / \mathrm{dt}$ and $\mathrm{di} / \mathrm{dt}$ during switching transients induce voltage spikes across the device parasitic inductances and capacitances, leading to resonances and under-damped oscillations. The consequent intense electromagnetic interferences may affect the correct functionality of the drive [4], [5]. A second important effect is due to voltage wave reflections across the cable that connects the converter to the motor, which are triggered by each PWM pulse supplying the machine [6]-[8]. As a consequence, severe voltage overshoots may arise at the motor terminals, which amplitude, depending on cable length and on the $\mathrm{dv} / \mathrm{dt}$, can be more than twice the inverter output voltage. Such voltage peaks at the motor terminals, which are usually followed by persistent ringing, represent the triggering event for partial discharges (PD), which are in turn the main cause of premature insulation breakdown [9]. The third phenomenon affecting drive reliability is due to the circulation of bearing currents in electric machines driven by HF converters. The inverter common-mode voltage is coupled to the machine bearing via parasitic capacitances. If the bearing electric field exceeds the dielectric strength of the insulating oil film on the bearing, a discharge current is produced that mechanically damages the bearing leading to premature failure [10].

With the purpose of properly investigate and describe the above-mentioned phenomena, it is necessary to recur to accurate high-frequency simulation models that consider the interaction between the distributed motor capacitances (i.e., winding to ground and/or turn-to-turn) and the HF supply from the converters. A variety of HF models of AC motors have been proposed over the last years in the literature. According to the approach used, electrical machine HF models can be roughly classified in three families [11]. Lumped-parameter circuit models [12]-[15] are equivalent circuit networks made of passive components, generally disposed as parallel RLC resonators and capacitive branches. These models make use of equivalent parameters to approximate the response of the machine in a wide frequency range. The values of the network components (i.e., resistors, inductors, and capacitors) are usually manually calculated from the values of specific points in the machine frequency characteristics. In [15] this time-consuming manual tuning is substituted by an automatic fitting procedure using a genetic optimization algorithm. Distributed-parameters circuit models [16], instead, model the machine winding in its length with per-unit circuit elements, generally a $\pi$-type or T-type 
equivalent. The aim is to obtain a better approximation of the winding parasitic parameters considering its structure and its interaction with the other machine parts. Although very good accuracy is achievable with this approach, the main drawback is the overall model complexity resulting in simulation time too large for their practical use. Transmission line models are the third family of HF motor models. They are based on the transmission line theory and analytically model the machine voltages and currents taking into account the effects of wave propagation through the winding. This approach, however, requires the use of time-consuming 3D finite element simulations with highly accurate geometries to correctly determine the inductance and capacitance matrices [17], [18].

The innovative contribution of this paper is twofold. On one hand, an accurate automatic modelling procedure is proposed, which is based on fitting the experimental data measured over a wide frequency range with a rational harmonic function. In particular, a genetic optimization algorithm (GA) is employed to find a harmonic function having variable poles and zeros that better approximates the machine frequency characteristics. By optimizing the polezero number and position, the model structure varies to better fit the particular machines that has to be modelled, increasing accuracy. Eventually, the optimized rational function can be then synthetized in its equivalent circuit. Furthermore, due to the high accuracy obtained with the proposed approach, a high-frequency model with time-varying poles and zeroes is developed to take into account the effects of the insulation degradation. To this purpose, three identical induction motors are subjected to an accelerated electrical aging procedure, and their frequency characteristics are measured at the healthy state and after each aging cycle. From the analysis of the results, it is possible to evaluate how the high-frequency model vary as the AC motor undergoes an electrical ageing. Such a model can be profitably used either to implement prognostic features in high-speed electrical drives or for design purposes.

This paper is organized as follows. Section II briefly describes the accelerated aging procedure that has been conducted on the induction machines under test. Section III focuses on the proposed modelling procedure. Finally, the fitting results are shown and discussed in the last section.

\section{AcCelerated Aging Tests}

Fig. 1 shows a picture of the experimental setup employed to perform the accelerated aging of the induction motors under test. The machines are three identical low-voltage induction motors rated $0.37 \mathrm{~kW}$, with class $\mathrm{F}$ insulation $\left(155^{\circ} \mathrm{C}\right)$. An inverter using SiC-MOSFETs is used to supply the motor through a long cable to enhance the voltage waveform reflection and achieve high overvoltage at the machine terminals. The inverter operates with a DC-link voltage of 540 $\mathrm{V}$ and a switching frequency of $32 \mathrm{kHz}$. A train of PWM pulses is applied to the machine phase terminals connected in parallel at a constant duty-cycle. Each aging cycle lasts for 8 hours, thus total of 922 million pulses are applied to each phase during each cycle. During the aging procedure, the machine current, voltage and the temperature on the case are monitored. In particular, since the test are performed with the intention of isolate the effects of the electrical phenomena from those due to the temperature, the RMS current absorbed by the motor is kept below the rated value while the machine is externally cooled with forced ventilation. The steady state temperature measured on the machine case after a few hours

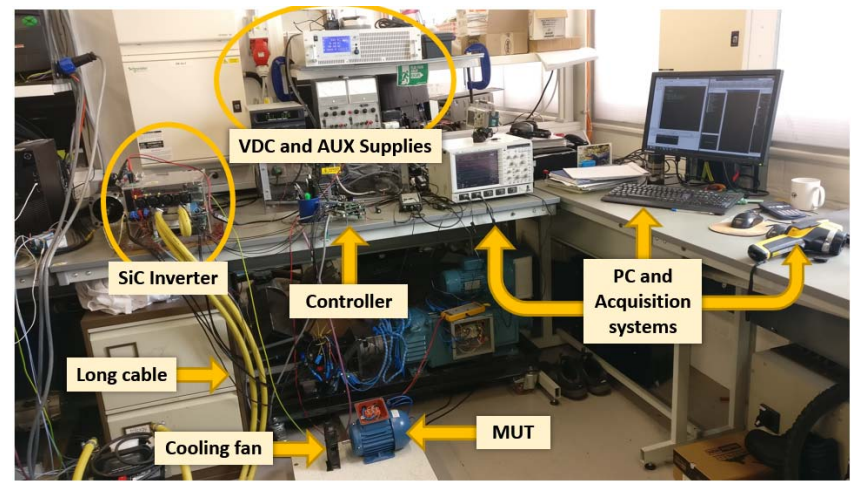

Fig. 1. Picture of the experimental setup for the accelerated electrical aging tests performed on the motor under test.

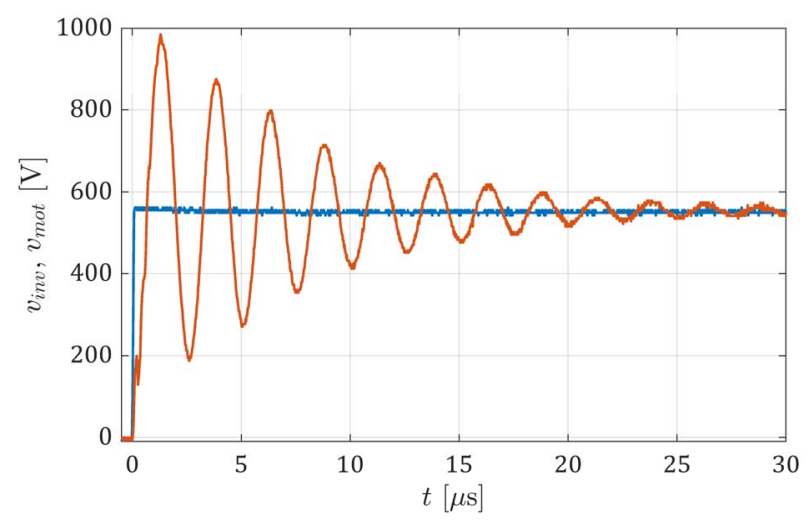

Fig. 2. Inverter output voltage (blue waveform) and overvoltage at the motor terminals (red waveform).

of ageing is around $40{ }^{\circ} \mathrm{C}$. The voltage waveforms at the inverter output and at motor terminals are shown in Fig. 2. The rise time of the inverter voltage pulse is around $70 \mathrm{~ns}$, producing a peak overvoltage on the motor of about $1 \mathrm{kV}$.

A high-precision impedance analyzer, the HP 4194A, has been employed to measure the machine impedance in a wide frequency range. It can span a frequency range between 100 $\mathrm{Hz}$ and $30 \mathrm{MHz}$, with high accuracy and repeatability of the measures. Two characteristic impedances can be defined according to the relative connection of the machine terminals to the instrument for the measure. The common mode impedance, $Z_{c m}$, is measured between the three phase terminals shorted together and the ground terminal, while the neutral is floating. The differential impedance, $Z_{d m}$, is measured between one of the phase terminals and the other two shorted together, with the neutral and the ground terminals floating. The common mode and differential impedances as a function of frequency are shown in Fig. 3 and Fig. 4, respectively for the healthy insulation state and for a degraded condition after a few aging cycles. Only one of the tested motors is shown in the figure, but similar results are obtained for the other two machines. The common mode impedance is characterized by a very high magnitude at low frequency, which is due to the blocking effect of the phase-toground capacitances generated by the insulation between the phase coils and the stator iron. The differential impedance, instead, has lower magnitude at low frequency and is characterized by two adjacent resonance peaks around 100 $\mathrm{kHz}$. A higher number of resonance peaks and notches with lower amplitude are observed in the very-high-frequency range for both the impedances. From a modelling point of view, each peak and notch suggest the presence, respectively, 

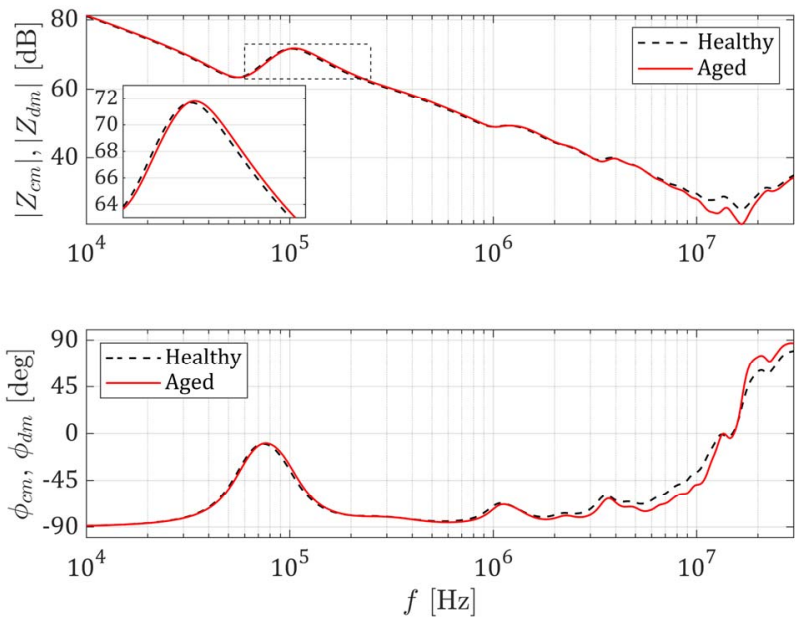

Fig. 3. Common mode impedance as a function of frequency, expressed as magnitude and phase. The black dashed line refers to the healthy insulation state, the red line to the measure taken after about 50 hours.
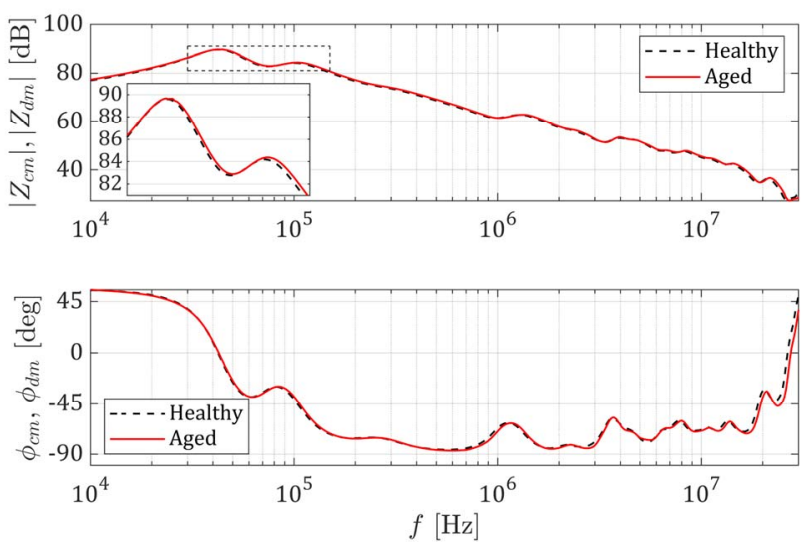

Fig. 4. Differential impedance as a function of frequency, expressed as magnitude and phase. The black dashed line refers to the healthy insulation state, the red line to the measure taken after about 50 hours..

of a complex conjugate pole or zero pair. Therefore, the number of pole-zero pairs increases at higher frequencies. This aspect can be considered in the automatic fitting procedure to reduce the computational burden and the time cost of the genetic algorithm. Regarding the aging effects, it appears that the impedance variations due to electrical stress are mainly concentrated in the very high-frequency portion of the spectrum and are more evident in the common mode impedance. The effects in the range between $10 \mathrm{kHz}$ and 1 $\mathrm{MHz}$ progress at a much slower rate than in the case of thermal aging, as shown in [19]. This could be due to the fact that, unlike partial discharges that are localized phenomena, the thermal stress affects greater portions of the winding, in a more uniform way. A more intense voltage level could probably speed up the aging process due to a more diffused partial discharge activity. However, the focus of the aging tests is the investigation of the aging progression in a low voltage drive operating in realistic conditions. The very small variations in the machine impedances require a very accurate fitting to proper model the aging progression.

\section{Automated Modelling Procedure}

The proposed modelling procedure has the objective to obtain the highest fitting accuracy as possible in order to track the small aging steps observed during the electrical aging campaign. Computational burden is also an important feature to reduce the modelling time. The main drawback of the models that are commonly used in the literature is the limited degree of freedom in adjusting the model parameters. A fixed model structure, in fact, can only achieve limited fitting accuracy, especially at higher frequency. To overcome this limitation, the proposed solution aims at finding the best rational harmonic function in the frequency domain so that the deviation between the measured and the simulated values of the impedance is minimized. Two different functions need be found, one to approximate the common mode impedance, denoted as $\tilde{Z}_{c m}$, and one for the differential impedance, indicated as $\tilde{Z}_{d m}$. The novel characteristic of this approach is that, by using a mathematical function to approximate the frequency response, the poles and zeroes are not predetermined in number and location as for a fixed circuit topology. A network representation of the model can then be obtained from the identified rational function.

A genetic optimization algorithm has been implemented to perform the automatic identification of the common mode and differential harmonic functions. A GA is an optimization routine based on the concept of natural selection, driving mechanism of biological evolution. In a GA, an initial population comprising a certain number of individuals is generated and let to evolve through the course of various generations. Each individual in the population is represented by a vector of optimization variables (genes), which can vary within a predefined search space. At each generation, the entire population is evaluated according to the criteria of a fitness function, and the individuals are sorted based on their result. At this point, the best performing individuals are selected for recombination, so that a new generation is produced (children) from the previous one (parents) through the crossover and the mutation operators. The process repeats until a stopping criterion is satisfied. This type of optimization approach can be very profitably used for problems with a large number of parameters, which in general are characterized by a significant number of local minima that prevent them to be solved by conventional algorithms. For this reason, it has been chosen in this work. In the following, the main aspects of the proposed algorithm are described, highlighting the features that allow a good accuracy to be reached.

\section{A. Fitness Function Definition}

The fitness function is the criterion that the GA uses to decide on the goodness of a certain individual among others. The fitness function that is used in the proposed algorithm is the sum of the two absolute errors calculated in terms of magnitude and phase for the two impedances to be fitted.

$$
F F=\sum_{k=1}^{N_{p t}} E_{c m}\left(f_{k}\right)+E_{d m}\left(f_{k}\right)
$$

In (1), $f_{k}$ is the $k^{\text {th }}$ measured frequency point, $N_{p t}$ is the total number of measured data for each impedance, $E_{c m}$ is the value of the absolute error calculated for the common-mode impedance, and $E_{d m}$ the one calculated for the differential impedance:

$$
\begin{aligned}
E_{j}\left(f_{k}\right)=\operatorname{abs}\left(\left|Z_{j}\left(f_{k}\right)\right|-\left|\tilde{Z}_{j}\left(f_{k}\right)\right|\right) \\
+a b s\left(\Varangle Z_{j}\left(f_{k}\right)-\Varangle \tilde{Z}_{j}\left(f_{k}\right)\right)
\end{aligned}
$$

where $j$ represents either $\mathrm{cm}$ or $d m$, and the " $\sim$ " symbol denotes the approximation functions. To be sure to obtain a well scaled fitness function, in (2), the magnitude of the 
impedance is expressed in $\mathrm{dB}$ and the phase in degrees. In this way, both errors are equally weighted during the optimization leading to a faster and more accurate convergence of the algorithm.

\section{B. Harmonic Function Form and Search Space Boundaries}

The harmonic functions to be found that approximate the machine impedances are implemented in the algorithm in the poles-zeroes form as in (3).

$$
\tilde{Z}=K \frac{\prod_{p=1}^{N_{z r}}\left(s-\omega_{p}\right) \prod_{q=1}^{N_{z c c}}\left(\frac{s^{2}}{\omega_{n, q}}+\frac{2 \delta_{v}}{\omega_{n, q}} s+1\right)}{s^{\mu} \prod_{t=1}^{N_{p r}}\left(s-\omega_{t}\right) \prod_{v=1}^{N_{p c c}}\left(\frac{s^{2}}{\omega_{n, v}}+\frac{2 \delta_{v}}{\omega_{n, v}} s+1\right)}
$$

In the above equation, $K$ is the function gain, $N_{p r}, N_{z r}, N_{p c c}$, and $N_{z c c}$ are, respectively, the number of real poles, real zeroes, and complex conjugate pairs of poles and zeroes. $\omega_{p}$ and $\omega_{t}$ are the frequency locations, in $\mathrm{rad} / \mathrm{s}$, of the real zeroes and poles, while each complex conjugate pair of poles or zeroes is described by its natural pulsation $\omega_{n}$ and the damping factor $\delta . \mu$ is the algebraic multiplicity of the poles in the origin. Its value is set to 1 for the common-mode impedance and to 0 for the differential impedance.

By employing the description in (3) for the harmonic functions to be optimized, defining the individuals' optimization variables becomes straightforward. The vector $x$ of the optimization variables is therefore defined as follows:

$$
x=\left[\boldsymbol{\Omega}_{p R} \boldsymbol{\Omega}_{z R} \boldsymbol{\Omega}_{p C C} \boldsymbol{\Delta}_{p C C} \boldsymbol{\Omega}_{z C C} \boldsymbol{\Delta}_{z C C} K\right]
$$

In (4):

- $\boldsymbol{\Omega}_{p R} \in \mathbb{R}^{1 \times N_{p r}}$ is the sub-vector of the real poles;

- $\boldsymbol{\Omega}_{z R} \in \mathbb{R}^{1 \times N_{z r}}$ is the sub-vector of the real zeroes;

- $\boldsymbol{\Omega}_{p C C}, \boldsymbol{\Delta}_{p C C} \in \mathbb{R}^{1 \times N_{p c c}}$ are the vectors of the natural pulsations and damping factors of the complex poles;

- $\boldsymbol{\Omega}_{z C C}, \boldsymbol{\Delta}_{z C C} \in \mathbb{R}^{1 \times N_{z c c}}$ are the vectors of the natural pulsations and damping factors of the complex zeroes;

- $\quad K \in \mathbb{R}$ is the gain.

Defining the individual as descripted above makes easier to control both the generation of the initial population, which has been found to be a key element for the fast and accurate convergence of the algorithm, and the selection of the constraints for the search space. The latter advantage is due to the knowledge of the shape of the frequency curves to be fitted, so that the poles and zeroes can be limited to the frequency range of interest (i.e., between $10 \mathrm{kHz}$ and 30 $\mathrm{MHz}$ ). furthermore, the variation of the damping factors is naturally limited between 0 and 1 . The only element that makes it difficult to define limits for is the function gain $K$. The reason is that its value can have a very large variation range, therefore a few tries are needed to find the proper boundaries for this parameter, before starting the core optimization procedure. This step does not require too many generations to be processed, since after a few iterations the algorithm tends to converge towards a value that is close to the final one. A starting procedure could also be implemented to automatize this step.

\section{Generation of the Initial Population}

A crucial factor in determining the convergence of the genetic algorithm is the generation of the initial set of individuals to start the evolutionary process. It has, in fact, been observed that uniformly generating the initial population allocating the poles and zeroes in the frequency range of interest leads to the non-convergence of the algorithm in a reasonable time. To overcome this issue, a specialized generation function has been built that divides the frequency range to be fitted in three intervals, according to the concentration of resonance peaks in the measured data. The first interval is defined between 10 and $100 \mathrm{kHz}$, with a lower number of pole-zero pairs; the second one is defined from 100 $\mathrm{kHz}$ to $1 \mathrm{MHz}$, presenting an higher number of peaks; The last interval goes from $1 \mathrm{MHz}$ to $30 \mathrm{MHz}$ and presents the higher concentration of resonances and notches. For each of the three intervals a weighting factor is selected so that their sum is equal to unity (e.g., $w_{1}+w_{2}+w_{3}=1$ ). The number of poles to be allocated in each frequency segment is then equal to the total number of poles or zeroes multiplied by the associated weight. The poles and zeroes are distributed uniformly in each interval also considering an overlap factor. The latter enlarge each frequency interval by a certain percentage to achieve an initial population with a higher variety. A good value for the overlap factor is found to be between $15 \%$ and $20 \%$. With the above-described generation function, both speed and accuracy of the genetic algorithm are greatly improved.

\section{Selection, Crossover and Mutation Operators}

The crossover operator is used after the individuals have been evaluated according to the fitness function and a percentage of them has been selected for recombination. The selection function used in this work is the stochastic uniform sampling (SUS). In SUS, the selection of the parents is made by sampling at an equal rate along a line made by a number of segments that is equal to the number of individuals evaluated. Each segment of the line is proportional to the fitness value of the associated individual. By sampling along the line at a constant rate, the better individuals are more favourably selected with respect to the weaker ones.

The intermediate crossover function is used in the proposed algorithm. This crossover operator creates a child $c$ as the weighted average of its parents $p_{1}$ and $p_{2}$.

$$
c=p_{1}+R *\left(p_{2}-p_{1}\right)
$$

$R$ is a random real number, whose variation range controls the position of the child with respect to its parents. If $R \in[0 ; 1]$, then the child lays between the values of the genes of its parents. In the proposed GA implementation, $R$ is set to vary up to 1.5 , meaning that the value calculated for the child can be outside the segment defined by its parents. This choice, despite being theoretically in contrast with the convergence of the optimization algorithm, has been found to be beneficial for accuracy, since, together with the mutation operator, gives more flexibility in the search space. Two other important parameters in the crossover function are the so-called elite fraction (set to 0.05) and crossover fraction (set to 0.1). The first specifies the percentage of individuals that will survive to the next generation, while the latter specifies the percentage of children that will be created by crossover. Both these parameters let the algorithm to maintain both the best and the worst individuals of a generation to the new one. This can be useful to avoid premature convergence to a local minimum. 
The mutation crossover applies to the children obtained by crossover a small random change in their gene values. In this way, the algorithm can investigate a broader space and achieve more genetic diversity. The selected operator for the proposed GA is the uniform mutation. First, part of the genes of each individual are selected for mutation with a certain probability rate (i.e., $15 \%$ for our case), then the value of the selected genes is replaced by a random value within the constraints that are set for that variable.

\section{E. Local Optimization}

As it can be deduced by the previous description, a series of parameter are included in the proposed algorithm that increase the genetic diversity of the population. Furthermore, the size of the population itself is chosen to be fifteen times the length of the optimization variables vector, which is a large number considering the high order of the rational function to properly identify the high-frequency points. This is due to the large number of local minima that are present in the search space, because of the complexity of the problem and the large number of pole-zero pairs. With the values indicated above for the crossover rate $R$, for the elite and crossover fractions, and for the mutation rate, a good genetic diversity is reached, and the procedure can converge near the global minima. However, for the same reason, it is then hard for the algorithm to properly optimize toward the best fit within the found minimum. It is for this reason that, after the GA run is completed, the optimized set of variables is used as the initial solution of a constrained minimization problem to be solved with a gradient-base routine, which is best suited for local minima optimizations. The selected algorithm is the interiorpoint optimization. The function value is reduced up to one third of its starting value by using this approach, which leads to a very good final accuracy.

Speaking about execution time of the whole algorithm, the GA take between 15-20 minutes to complete on a quad-core PC with 16 GB RAM, while the local minimization requires about 1-2 minutes to converge. As a result, the total required time is lower than 25 minutes for the fitting of the two impedances of a motor, confirming the goodness of the proposed approach.

\section{RESULTS AND DISCUSSION}

Fig. 5 shows the result of the fitting procedure on the magnitude of the common-mode and differential impedances at the healthy insulation state. The phase is depicted in Fig. 6. There is a very good agreement between the measured and the fitted curves, despite minor divergences at the limits of the frequency interval in both impedances. To quantify the accuracy of the algorithm the mean absolute error is calculated for both impedances as the averaged sum of the deviations at each measured frequency. Its value is equal to $0.41 \mathrm{~dB}$ in the case of the modules and degrees for the phases). For the common-mode impedance, the order of the harmonic function is 20, while for the differential impedance is 22 ( 2 more poles in the lower frequency interval). To improve the algorithm accuracy at the boundaries of the frequency window a few more measurement point could be needed, however, the major features are captured in this configuration, since the low frequency behaviour is not an objective of the model.

To verify the goodness of the algorithm in catching the small variation due to the aging, a comparison between two aging states is depicted in Fig. 7 and Fig. 8 for the commonmode and the differential impedances, respectively. The

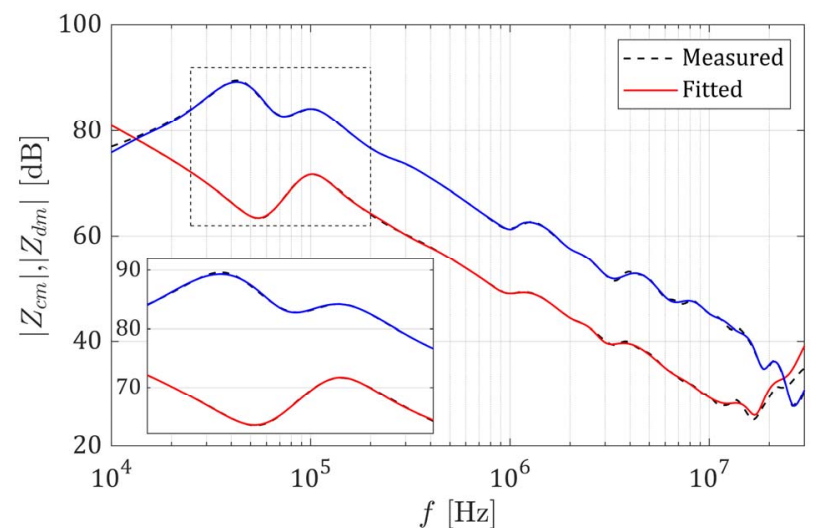

Fig. 5. Comparison of fitting accuracy between healthy and aged state for the common-mode impedance.

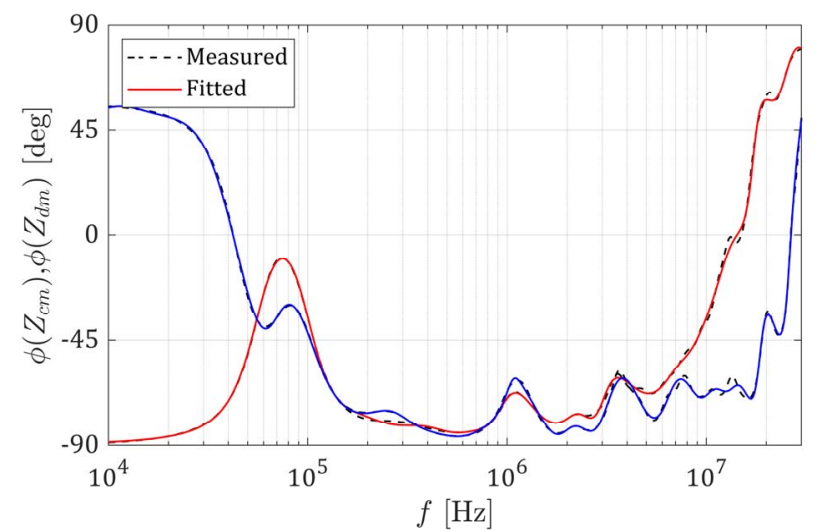

Fig. 6. Comparison of fitting accuracy between healthy and aged state for the differential impedance.

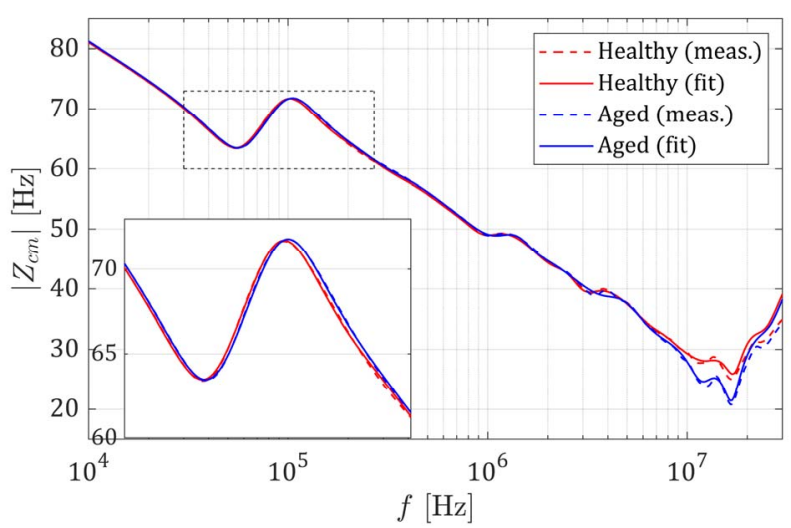

Fig. 7. Fitting accuracy to distinguish between different aging steps (common-mode impedance).

\section{TABLE I. TABLE TITLE}

\begin{tabular}{|c|c|c|}
\hline & $\begin{array}{c}\text { Common-mode } \\
\text { imp. }\end{array}$ & Differential imp. \\
\hline Healthy - Aged (exp.) & 1.98 & 0.68 \\
\hline Meas. - Fit. (Healthy) & 0.41 & 0.41 \\
\hline Meas. - Fit (Aged) & 0.49 & 0.46 \\
\hline
\end{tabular}

values of the mean absolute errors are indicated in Table I both for the experimental data (deviation between healthy and aged condition) and for the fitting. The accuracy obtained with the proposed procedure is good enough to catch aging variations of the entity observed in this work. By performing more tests 


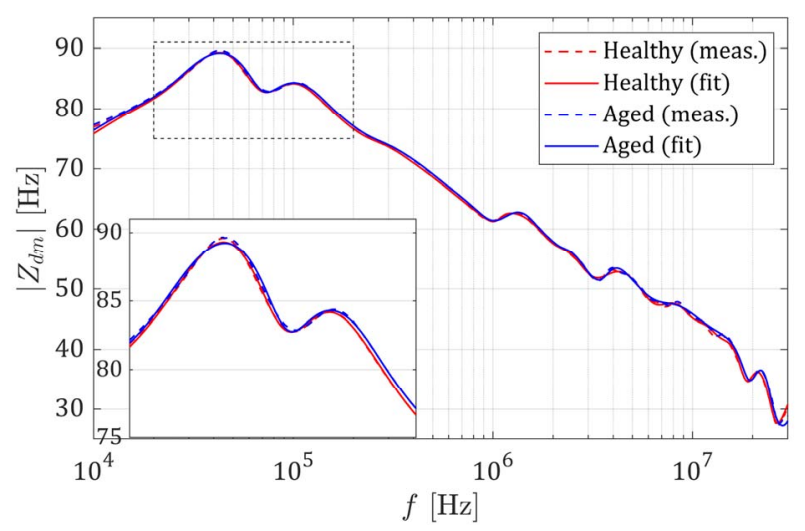

Fig. 8. Fitting accuracy to distinguish between different aging steps (differential impedance).

on the motors, it could be then possible to build an accurate high-frequency model with time-varying parameters. This model could be profitably used either for design purposes or in model-based diagnosis algorithms. For the latter, a greater number of tests would be needed also to add statistical features to the model and to characterize different motor sizes and winding topologies.

\section{SUMMARY AND CONCLUSIONS}

In this paper, an accurate automated fitting procedure is proposed for the modelling of AC machines in the highfrequency domain. The procedure is based on the optimization, using a genetic algorithm, of a rational harmonic function in the poles-zeroes form. Thanks to the analytical form of the problem, the algorithm convergence is fast even when a large population is analysed. The accuracy achieved with the proposed procedure let to distinguish between small variation in the measured characteristic impedances of the machine. Such small variations are, for example, those observed during an accelerated electrical aging campaign performed on the machine tested for the study. The proposed approach could be used both for high-frequency drive design purposes and to include model-based diagnostic features to the electrical drive.

\section{REFERENCES}

[1] D. Han, Y. Li and B. Sarlioglu, "Analysis of $\mathrm{SiC}$ based power electronic inverters for high speed machines," 2015 IEEE Applied Power Electronics Conference and Exposition (APEC), Charlotte, NC, 2015, pp. 304-310.

[2] H. Kim, M. W. Degner, J. M. Guerrero, F. Briz and R. D. Lorenz, "Discrete-Time Current Regulator Design for AC Machine Drives," in IEEE Transactions on Industry Applications, vol. 46, no. 4, pp. 14251435, July-Aug. 2010.

[3] D. Gerada, A. Mebarki, N. L. Brown, C. Gerada, A. Cavagnino and A. Boglietti, "High-Speed Electrical Machines: Technologies, Trends, and Developments," in IEEE Transactions on Industrial Electronics, vol. 61, no. 6, pp. 2946-2959, June 2014

[4] I. Josifović, J. Popović-Gerber and J. A. Ferreira, "Improving SiC JFET Switching Behavior Under Influence of Circuit Parasitics," in IEEE
Transactions on Power Electronics, vol. 27, no. 8, pp. 3843-3854, Aug. 2012.

[5] D. Han and B. Sarlioglu, "Study of the switching performance and EMI signature of SiC MOSFETs under the influence of parasitic inductance in an automotive DC-DC converter," 2015 IEEE Transportation Electrification Conference and Expo (ITEC), Dearborn, MI, 2015, pp. 1-8.

[6] A. Cavallini, D. Fabiani and G. C. Montanari, "Power electronics and electrical insulation systems 8 Part 1: Phenomenology overview," in IEEE Electrical Insulation Magazine, vol. 26, no. 3, pp. 7-15, MayJune 2010.

[7] M. J. Melfi, "Low-Voltage PWM inverter-fed motor insulation issues," in IEEE Transactions on Industry Applications, vol. 42, no. 1, pp. 128133, Jan.-Feb. 2006.

[8] C. Petrarca, A. Maffucci, V. Tucci and M. Vitelli, "Analysis of the voltage distribution in a motor stator winding subjected to steep-fronted surge voltages by means of a multiconductor lossy transmission line model," in IEEE Transactions on Energy Conversion, vol. 19, no. 1, pp. 7-17, March 2004

[9] G. C. Stone, I. Culbert, E. A. Boulter, and H. Dhirani, "Evaluating insulation materials and systems," in Electrical Insulation for Rotating Machines: Design, Evaluation, Aging, Testing, and Repair, 2th ed. Piscataway, NJ, USA: Wiley-IEEE Press, 2014, ch. 2, sec. 1, pp. 4954.

[10] M. Schuster and A. Binder, "Comparison of different inverter-fed AC motor types regarding common-mode bearing currents," 2015 IEEE Energy Conversion Congress and Exposition (ECCE), Montreal, QC, 2015, pp. 2762-2768.

[11] M. Asefi and J. Nazarzadeh, "Survey on high-frequency models of PWM electric drives for shaft voltage and bearing current analysis," in IET Electrical Systems in Transportation, vol. 7, no. 3, pp. 179-189, 9 2017.

[12] C. Bi et al., "High-frequency electric-motor modelling for conducted CM current prediction in adjustable speed drive system," in IET Power Electronics, vol. 11, no. 7, pp. 1257-1265, 1962018.

[13] A. Boglietti, A. Cavagnino and M. Lazzari, "Experimental HighFrequency Parameter Identification of AC Electrical Motors," in IEEE Transactions on Industry Applications, vol. 43, no. 1, pp. 23-29, Jan.feb. 2007.

[14] G. Vidmar and D. Miljavec, "A Universal High-Frequency ThreePhase Electric-Motor Model Suitable for the Delta- and Star-Winding Connections," in IEEE Transactions on Power Electronics, vol. 30, no. 8, pp. 4365-4376, Aug. 2015.

[15] M. Degano, P. Zanchetta, L. Empringham, E. Lavopa and J. Clare, "HF induction motor modeling using automated experimental impedance measurement matching," in IEEE Transactions on Industrial Electronics, vol. 59, no. 10, pp. 3789-3796, Oct. 2012.

[16] J. Sun and L. Xing, "Parameterization of Three-Phase Electric Machine Models for EMI Simulation," in IEEE Transactions on Power Electronics, vol. 29, no. 1, pp. 36-41, Jan. 2014.

[17] Y. Ryu, B. Park and K. J. Han, "Estimation of High-Frequency Parameters of AC Machine From Transmission Line Model," in IEEE Transactions on Magnetics, vol. 51, no. 3, pp. 1-4, March 2015.

[18] H. De Gersem and A. Muetze, "Finite-Element Supported Transmission-Line Models for Calculating High-Frequency Effects in Machine Windings," in IEEE Transactions on Magnetics, vol. 48, no. 2, pp. 787-790, Feb. 2012.

[19] C. Zoeller, M. A. Vogelsberger and T. M. Wolbank, "Assessment of insulation condition parameters of low-voltage random-wound electrical machine," IECON 2016 - 42nd Annual Conference of the IEEE Industrial Electronics Society, Florence, 2016, pp. 1470-1475. 